\title{
CONF- $961086--5$
}

Performance Tradeoffs for a Surface Micromachined Microengine

S. L. Miller, J. J. Sniegowski, G. LaVigne, and P. J. McWhorter

\author{
Sandia National Laboratories \\ Mail Stop 1080 \\ P.O. Box 5800 \\ Albuquerque, NM 87185-1080
}

\begin{abstract}
An electromechanical model of Sandia's microengine is developed and applied to quantify critical performance tradeoffs. This is done by determining how forces impact the mechanical response of the engine to different electrical drive signals. To validate the theoretical results, model-based drive signals are used to operate actual engines, where controlled operation is achieved for the following cases: 1) spring forces are dominant, 2) frictional forces are dominant, 3) linear inertial forces are dominant, 4) viscous damping forces are dominant, and 5) inertial load forces are dominant. Significant improvements in engine performance are experimentally demonstrated in the following areas: positional control, start/stop endurance, constant speed endurance, friction load reduction, and rapid actuation of inertial loads.
\end{abstract}

Keywords: microengine, actuator, modeling

\section{Introduction}

The microengine developed at Sandia National Laboratories, shown in Fig. 1, produces rotational motion of an output gear that can deliver torque to other mechanisms of interest. ${ }^{1-5}$ Mechanisms successfully driven by the surface micromachined engine include an optical shutter, torque-increasing gear reduction units, and linear racks. While functionality has been demonstrated, the ultimate goal is to achieve optimum performance of the engine in practical actuator applications, through quantitatively understanding performance tradeoffs.

The nature of a particular application determines the specification of optimum performance. One may need, for example, to minimize friction, minimize the drive voltage amplitude, minimize joint stress, maximize angular acceleration, and/or precisely achieve some specified time-dependent position of a load. Performance tradeoffs include answering such questions as: how does the acceleration rate or top speed of the engine impact the necessary drive signal amplitude; how controlled must the drive signal be to reduce frictional forces to an acceptable level; for a given drive signal amplitude, how does the top speed of the engine depend on the viscosity of the medium in which it operates; is it worth implementing complex drive signals to reduce stress associated with abruptly stopping the engine. A valid electromechanical model of the microengine is needed both to achieve optimum performance and to enable a designer or end user to make critical performance tradeoffs.

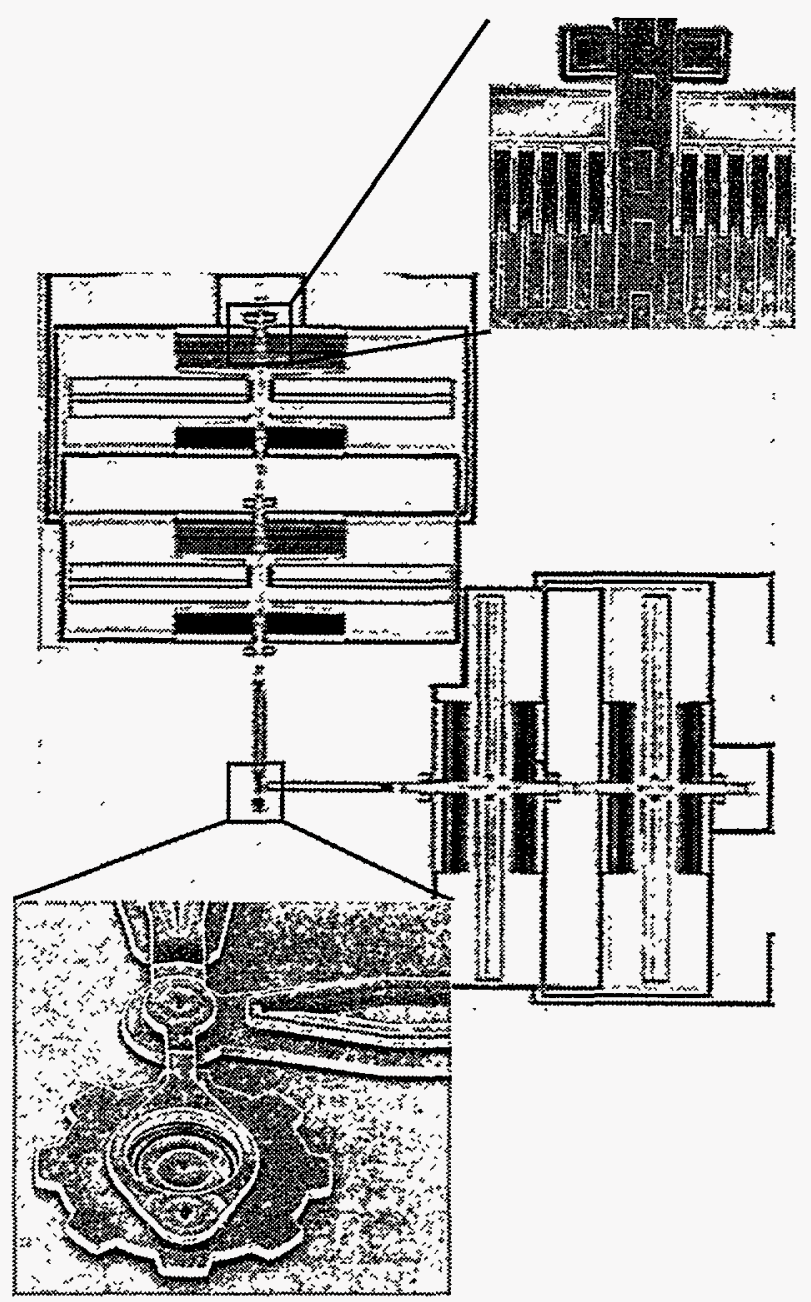

Fig. 1. The microengine consists of orthogonally oriented linear comb drive actuators mechanically connected to a rotating gear capable of providing torque to a load device. 


\section{DISCLAIMER}

Portions of this document may be illegible in electronic image products. Images are produced from the best available original document. 


\section{DISCLAIMER}

This report was prepared as an account of work sponsored by an agency of the United States Government. Neither the United States Government nor any agency thereof, nor any of their employees, makes any warranty, express or implied, or assumes any legal liability or responsibility for the accuracy, completeness, or usefulness of any information, apparatus, product, or process disclosed, or represents that its use would not infringe privately owned rights. Reference herein to any specific commercial product, process, or service by trade name, trademark, manufacturer, or otherwise does not necessarily constitute or imply its endorsement, recommendation, or favoring by the United States Government or any agency thereof. The views and opinions of authors expressed herein do not necessarily state or reflect those of the United States Government or any agency thereof. 
A model that is useful to evaluate performance tradeoffs is one that is predictive, and whose predictions are verifiable. To implement such a model requires sufficiently accurate knowledge of the physical parameters of the system. Though this can be relatively straightforward for macroscopic electromechanical systems, it certainly is not for microscopic systems such as the microengine. For example, measuring viscous damping, frictional, spring, and electrostatic forces in devices with features on the order of microns in size requires special care, and often indirect methods.

In this paper we develop and apply an electromechanical performance model to quantify performance tradeoffs of Sandia's microengine. The model is used to understand what physical quantities impact performance, and in what way. Model-based drive signals are used to successfully induce and achieve desired engine operation for the following cases: 1) spring forces are dominant, 2) frictional forces are dominant, 3) linear inertial forces are dominant, 4) viscous damping forces are dominant, and 5) inertial load forces are dominant. Significant performance improvements resulting from the performance analysis are experimentally demonstrated.

\section{Performance Model}

The dominant forces that impact the operation of the microengine are incorporated in the performance model. These include electrostatic forces acting on the

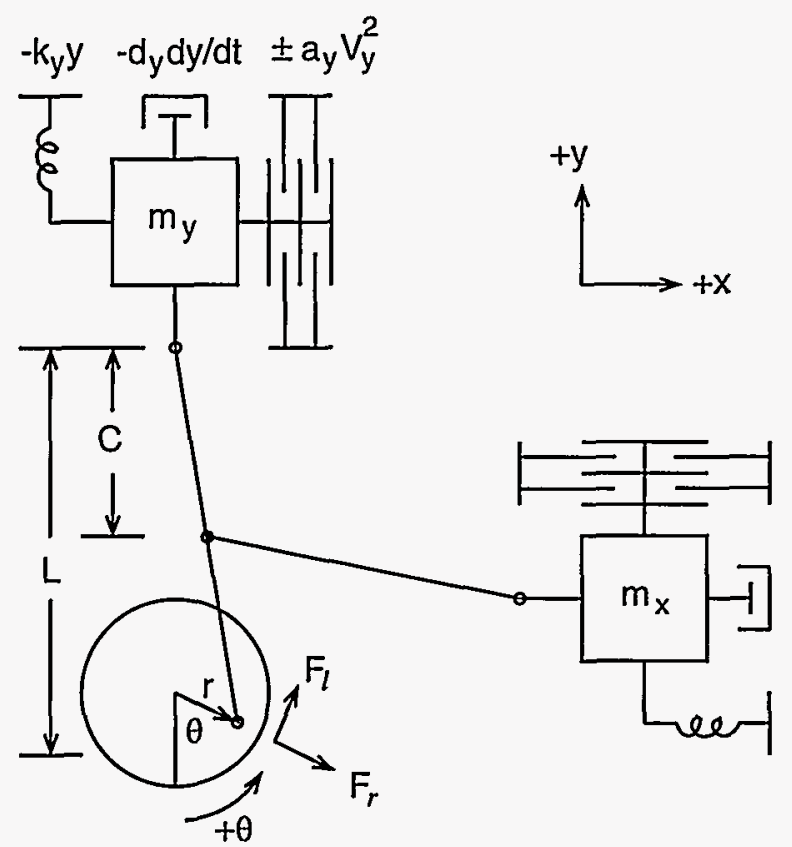

Fig. 2. The comb drives experience forces due to supporting springs, air damping, electrostatic attraction, and the mechanical linkages connecting them to the gear. linear electrostatic actuators that drive the output gear, restoring forces due to the springs supporting the linear actuators, inertial forces associated with the linear motion of the electrostatic actuator elements, viscous damping forces due to the movement of the structure in air or in lubricating liquids such as oil, frictional forces due to the output gear rubbing on its hub, and inertial or other forces associated with loads being driven by the microengine.

The performance model is based on the schematic of the microengine shown in Fig. 2. The force between the gear and the linkage mechanism is resolved into radial and tangential components. The electrostatic, restoring spring, and viscous damping forces in the $y$ direction are expressed as:

$$
\begin{gathered}
F_{y}= \pm a_{y} V_{y}^{2} \\
F_{y}=-k_{y} y \\
F_{y}=-d_{y} \frac{d y}{d t} .
\end{gathered}
$$

where $a_{y}$ is the electrostatic force constant, $k_{y}$ is the restoring spring force constant, and $d_{y}$ is the coefficient of viscous damping. Similar forces exist in the $x$ direction. To simplify the form of the resulting equations, we define the following terms:

$$
\begin{aligned}
\delta_{x} & =\frac{d_{x}}{2 m_{x}} \\
\omega_{x} & =\sqrt{\frac{k_{x}}{m_{x}}} \\
\gamma & =\frac{C}{L},
\end{aligned}
$$

where $m_{x}$ is the mass of the structure moving in the $x$ direction, $\delta_{x}$ is the viscous damping ratio, $\omega_{x}$ the resonant frequency of the moving mass, and $C$ and $L$ are geometrical quantities illustrated in Fig. 2. Expressions similar to Eqs. (4) and (5) also exist for the $y$ direction of motion. The effects of the small angle deflections of the linkages are relatively insignificant, and hence are neglected. Omitting the details of the derivation, we solve Newton's equation $\Sigma F=m a$ for both $m_{x}$ and $m_{y}$, and obtain

$$
\begin{aligned}
V_{x}(\theta(t))= & \pm\left\{\frac { 1 } { \gamma } \frac { k _ { x } r } { a _ { x } } \left(\frac{\gamma^{2}}{\omega_{x}^{2}}\left[\left(\ddot{\theta}+2 \delta_{x} \dot{\theta}\right) \cos (\theta)-\dot{\theta}^{2} \sin (\theta)\right]\right.\right. \\
& \left.\left.+\left(\frac{F_{r}}{k_{x} r}+\gamma^{2}\right) \sin (\theta)+\frac{F_{l}}{k_{x} r} \cos (\theta)\right)\right\}^{\frac{1}{2}}
\end{aligned}
$$

and 


$$
\begin{aligned}
V_{y}(\theta(t)) & = \pm\left\{\frac { k _ { y } r } { a _ { y } } \left(\frac{1}{\omega_{y}^{2}}\left[\left(\ddot{\theta}+2 \delta_{y} \dot{\theta}\right) \sin (\theta)+\dot{\theta}^{2} \cos (\theta)\right]\right.\right. \\
& \left.\left.+1-\left(\frac{F_{r}}{k_{y} r}+1\right) \cos (\theta)+\frac{F_{l}}{k_{y} r} \sin (\theta)\right)\right\}^{\frac{1}{2}} .
\end{aligned}
$$

The motion of the microengine is related to the relevant forces by Eqs. (7) and (8). Specifically, they give the drive voltages $V_{x}(\theta(t))$ and $V_{y}(\theta(t))$ required to achieve some specified time-dependent angular position $\theta(t)$ when the forces are known. Methods to independently measure the forces and force constants required to evaluate these equations are given elsewhere. ${ }^{6}$ For the designs investigated here, the fact that $k_{x} \approx k_{y}, \omega_{x} \approx \omega_{y} a_{x} \approx a_{y}$, and $\delta_{x} \approx \delta_{y}$ simplifies the process of determining the force constants.

Drive signals created using Eqs. (7) and (8) are termed "model-based drive signals", and are used to achieve open loop control of the engine. The performance of the engine, i.e. its actual response to drive signals, depends on the nature of the various force contributions.

\section{Performance Tradeoffs}

Performance trade-offs are made by understanding how forces impact the response of the engine to drive signals. We now evaluate the impact of different force contributions on the response of the engine. In particular, we consider the various terms comprising Eqs. (7) and (8). We examine forces with different origins in the order they are listed in Table 1 . The fundamental results are illustrated with experimental data obtained from operating engines.

\subsection{Spring Forces}

The restoring forces of the springs supporting the moveable comb drive exist in all regimes of operation. In the absence of friction, damping, inertia, and load forces, the minimum possible voltage at which the engine can operate is the voltage required to overcome the maximum spring force. This occurs at $\theta=180^{\circ}$, where the deflection in the $y$ direction is $2 k r$. The voltage required to achieve this deflection is $V=$ $(2 \mathrm{kr} / \mathrm{a})^{1 / 2} \sim 65 \mathrm{~V}$ for the present design. The minimum operating voltage can be reduced either by reducing the spring constant $k$ or increasing the electrostatic force constant $a$. Reducing the radius $r$ at which the linkage is attached to the output gear will reduce the minimum operating voltage, but will also reduce the torque that can be delivered to a load.

A convenient reference by which to measure the forces in the microengine system is the restoring force when the springs are deflected one radius: $k r \sim 1.2 \mu \mathrm{N}$ in the present design. The magnitude of other forces will be referenced to this force throughout the remainder of the paper.

\subsection{Frictional Forces}

The forces $F_{r}$ and $F_{l}$ acting at the pin joint between the gear and the linkage can originate from several sources. Friction between the gear and hub can result in a nonzero tangential load force $F_{l}$. Also, when one is accelerating an inertial load, such as a large rotating disk, the torque required to quickly accelerate it results in a nonzero force $F_{l}$. Rotational inertia will be discussed in section 3.4.

A method to experimentally measure the frictional force between the gear and hub has been discussed elsewhere. ${ }^{6}$ It is found that for relatively low radial forces $\left(F_{r}<4 \mu \mathrm{N}\right)$, friction exhibits conventional behavior. Specifically $F_{l}=\mu F_{r}$ where $\mu \sim 0.5$. Using model-based drive signals (Eqs. (7) and (8)) with accurate physical parameters, the engine can be operated in regimes where the contribution to the force acting at the pin joint due to friction is much less than the spring force $k r$.

\begin{tabular}{|c|c|}
\hline $\begin{array}{l}\text { normalized } \\
\text { quantities* }\end{array}$ & $\begin{array}{l}\text { origins of } \\
\text { forces }\end{array}$ \\
\hline 1 & $\begin{array}{l}\text { spring forces } \\
\text { (support spring) }\end{array}$ \\
\hline$\frac{F_{r}}{k_{y} r}, \frac{F_{l}}{k_{y} r}$ & $\begin{array}{l}\text { frictional forces } \\
\text { (gear on hub) }\end{array}$ \\
\hline$\frac{\dot{\theta}^{2}}{\omega_{y}^{2}}, \frac{\ddot{\theta}}{\omega_{y}^{2}}$ & $\begin{array}{l}\text { inertial forces } \\
\text { (comb drive) }\end{array}$ \\
\hline$\frac{2 \delta_{y} \dot{\theta}}{\omega_{y}^{2}}$ & $\begin{array}{l}\text { viscous forces } \\
\text { (air, oil, etc.) }\end{array}$ \\
\hline$\frac{F_{r}}{k_{y} r}, \frac{F_{l}}{k_{y} r}$ & $\begin{array}{l}\text { load forces } \\
\text { (load inertia, } \\
\text { load friction) }\end{array}$ \\
\hline \multicolumn{2}{|c|}{$\begin{array}{l}\text { *To convert quantities in this } \\
\text { column into forces, multiply by } \\
k_{y} r=1.2 \mu \mathrm{N} \text {. }\end{array}$} \\
\hline
\end{tabular}

\section{Table 1: Force Terms}




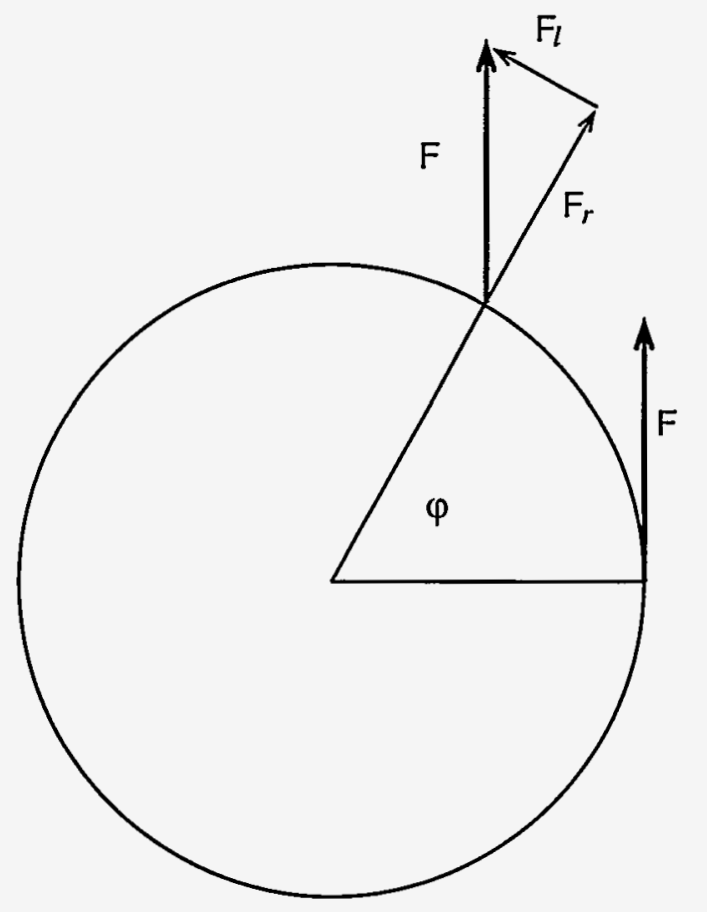

Fig. 3. The output gear of the engine self-adjusts such that the tangential component of the applied force $F$, referred to as the load force $F_{l}$, balances the frictional load torque. If the frictional force varies during rotation, the angle of advancement $\varphi$ also changes.

When the engine is operated without accurate model-based drive signals, the engine will "self-adjust" its phase relative to the drive signal in a way that depends on the frictional forces. Specifically, if an incorrect drive signal is used, the gear will advance through an angle $\varphi$ such that the tangential component of the force applied by the linear actuators balances the frictional load torque generated by the radial joint forces. This phenomenon is schematically illustrated in Fig. 3.

Experimental data from an engine exhibiting selfadjustment are shown in Fig. 4. The data shown are for an engine running at a constant speed of 25,000 revolutions per minute (rpm). The engine is first operated by applying a radial force $F_{r} / k r=4$ which results in significant frictional torque. To overcome this load, the torque term $F_{l} / k r$ in the model-based drive signals is gradually increased until the engine just starts to operate. This occurs at a value of $F_{l} / k r=1.75$; at lower values, the engine does not exhibit complete rotation. When the forces are properly balanced, at time $t=0$ the engine angle is $\theta=0$, as shown by the dots in Fig. 4. Next, the radial force is reduced to zero by setting $F_{r} / k r=0$ in the model-based drive signal equations, while at the same time keeping $F_{l} / \mathrm{kr}$ at the

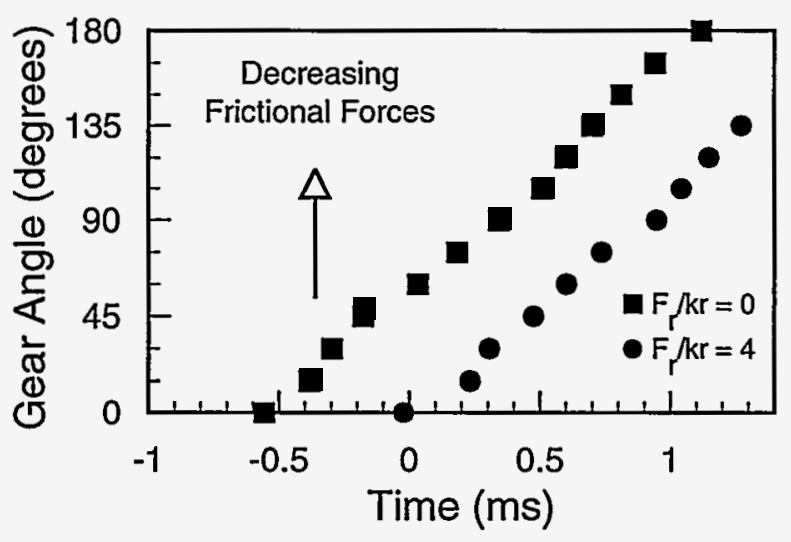

Fig. 4. Reducing the radial force $F_{r} / k r$ reduces the frictional force between the gear and hub. The decreased torque required to run the engine results in the gear advancing relative to the drive signal, as shown in Fig. 3.

original value of 1.75 . The modified drive signal results in lower friction than the first signal. As a result, the angle of the gear relative to the drive signal advances until the tangential component of the electrostatically applied force balances the reduced frictional load torque. Note that both the radial force and the frictional force vary simultaneously until equilibrium is reached with the drive signal. During the process of self adjustment the magnitude of the force applied to the gear through the pin joint remains approximately constant, but the angle at which the force is applied changes.

To achieve optimum performance, the force applied to the gear through the pin joint should be purely tangential. In this case, the entire drive force provides torque to the gear, and the absence of a radial force minimizes frictional forces. To accomplish this optimization using model-based drive signals, one should set $F_{r} / k r=0$ and the load force term as low as possible until the engine operates with a minimal angle of advancement. At this point, the applied force is determined by the load from the driven mechanism; the actual radial force, and hence frictional force, is minimized. Using this method, engine operation with load forces as low as $F_{l} / k r=0.1$ has been achieved when there is no load mechanism attached. This is more than an order of magnitude reduction in friction compared to that typically occurring when model-based drive signals are not used.

To summarize, there is a two-fold impact on performance as a result of self-adjustment to an incorrect drive signal. The angular position of the output gear will differ from the expected position. This can be important for applications where precise positioning of a load is important. The other impact is that the 


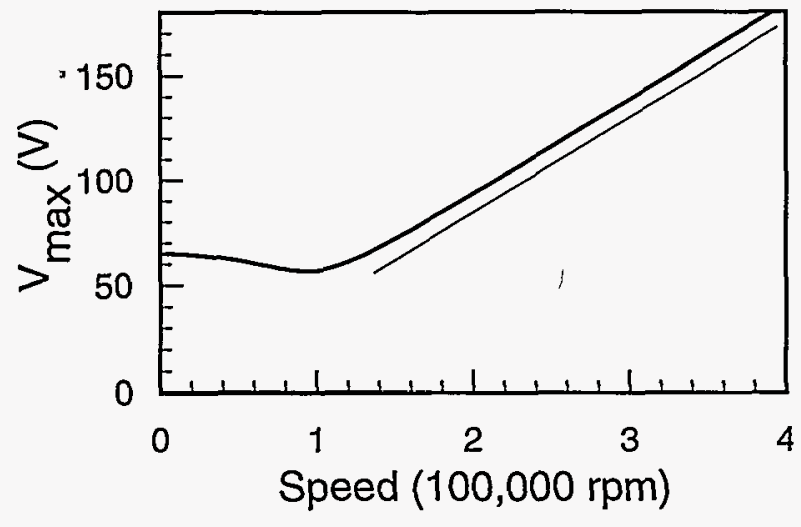

Fig. 5. The voltage required to operate the engine with minimal friction increases linearly with speed above the resonant frequency $(\sim 100,000 \mathrm{rpm}$.). The increase in voltage is due to the linear inertia of the comb drives.

frictional force is higher than necessary when the angle is advanced. Increased friction can potentially impact the reliability of the engine, and reduces the force that can be delivered to loads. If precise positioning and friction and wear reduction are not objectives relevant to a given application, the engine can easily be operated using simple periodic signals, such as sine or square waves.

\subsection{Linear inertia}

\subsubsection{Constant Speed}

At high speeds, significant forces (relative to $k r$ ) are associated with the linear inertia of the moving electrostatic comb drives. The resonant frequency $\omega_{y}$ for the engine is experimentally measured to be $\sim 1100$ $\mathrm{rad} / \mathrm{sec} \approx 1.6 \mathrm{kHz} \approx 100,000 \mathrm{rpm}$. Inertial forces become important when this speed is exceeded. To illustrate, we examine the minimum voltage required to operate the engine, which is the voltage peak in the $y$ direction resulting from Eq. (8). This voltage maximum is shown as a function of speed in Fig. 5. At low speeds, the voltage is limited by spring forces as discussed in the previous section. Near resonance, there is a slight dip. The voltage dip does not approach zero as it would for normal undamped resonant systems due to the asymmetry in the engine design. Above resonance, the voltage increases linearly with speed due to the dominance of the inertial term $(d \theta / d t)^{2}$.

In addition to the amplitude, the phase of the drive signal relative to the gear changes as the speed passes through the resonant frequency. The angle at which $V_{y}$ is maximum is given, in general, by

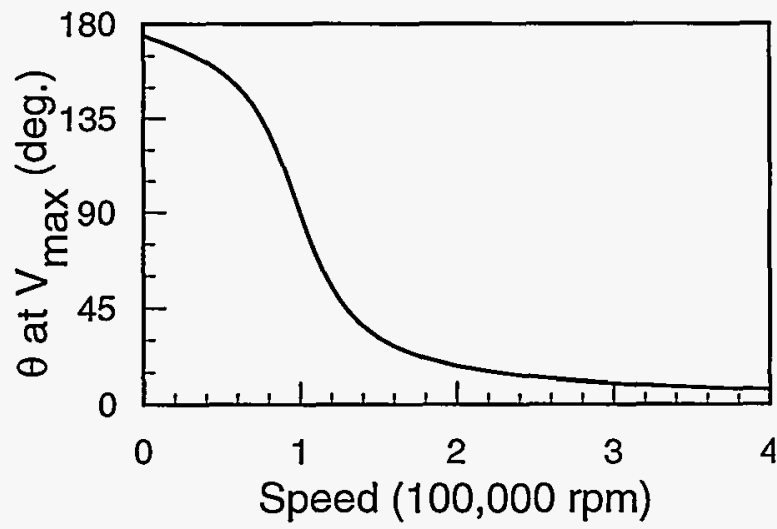

Fig. 6. The phase of the peak in the drive voltage shifts nearly $180^{\circ}$ as the engine speed passes through the resonant frequency. This is due to the linear inertia of the comb drives.

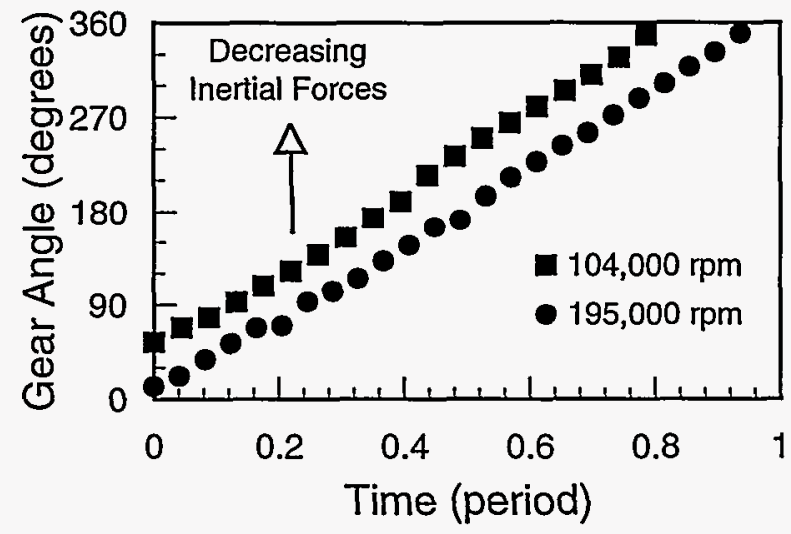

Fig. 7. The impact of inertia on engine performance is evident by operating the engine with drive signals that do not compensate for inertia. As the speed is reduced, inertial forces decrease, resulting in as significant phase shift of the engine. This response is qualitatively similar to the "self-adjustment" illustrated in Figs. 3 and 4.

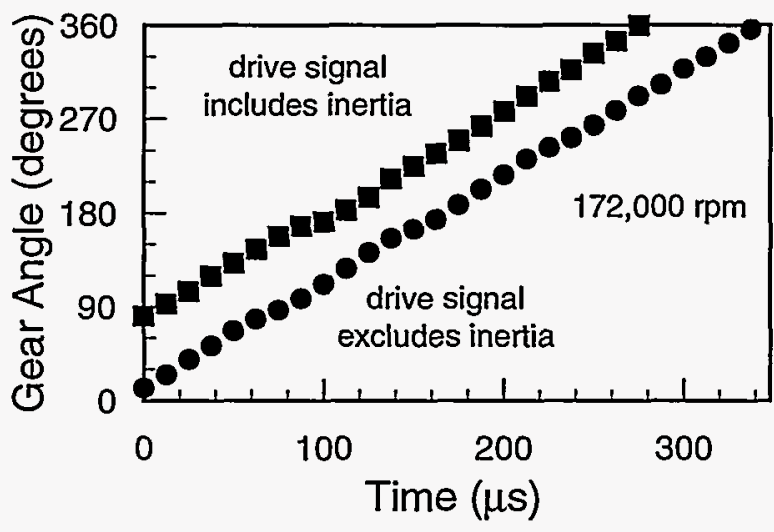

Fig. 8. Including inertial terms in the drive signal has the same effect on engine response as reducing the speed so that inertial forces are not large (compare with Fig. 7). 


$$
\tan \left(\left.\theta\right|_{V_{\max }}\right)=\frac{\frac{\ddot{\theta}}{\omega_{y}^{2}}+\frac{2 \delta_{y} \dot{\theta}}{\omega_{y}^{2}}+\frac{F_{l}}{k_{y} r}}{\frac{\dot{\theta}^{2}}{\omega_{y}^{2}}-\frac{F_{r}}{k_{y} r}-1} .
$$

The angle of maximum voltage is shown Fig. 6 as a function of engine speed. Note that the phase shifts nearly $180^{\circ}$. The phase shift is a direct consequence of the fact that the dominant effect of the electrostatic force is to accelerate the shuttle mass, i.e. inertial forces exceed spring forces.

This phase shift can have a significant impact on the performance of the engine. Suppose the engine is driven at a high speed with periodic signals, such as sine waves. Without the proper model-based drive signals, the electrostatically applied forces will not compensate for the inertial forces associated with the mass of the comb drives. Consequently, the engine system will selfadjust to provide the necessary inertial forces in the physically constrained system. These forces will be provided by the pin joint connecting the gear to the drive arm, and ultimately, by the hub on which the gear rotates. The excessive stress can potentially impact the reliability of the engine. An additional impact of the self-adjustment is the fact that the angular position of the gear could be significantly out of phase from the intended position.

These effects are graphically illustrated by the experimental data from a continuously running engine, shown in Fig. 7. An engine was operated at two different speeds with signals created using Eqs. (7) and (8), with the exception that the inertial terms were purposefully omitted. When the speed is increased from $104,000 \mathrm{rpm}$ to $195,000 \mathrm{rpm}$, the phase of the gear retreats via the self-adjustment phenomenon (compare with Fig. 6).

The effect of inertial forces is demonstrated an alternate way in Fig. 8. An engine is operated at a constant speed of $172,000 \mathrm{rpm}$ (well above the resonant frequency) with two different sets of drive signals. In one case, Eqs. (7) and (8) are used to create the drive signal with the inertial terms being included. In the second case, the inertial terms are omitted. It is clear that driving the engine at speeds where inertial forces are significant, without taking into account the inertial forces when creating the drive signals, results in significant self-adjustment of the phase of the engine. When the engine self-adjusts, excessive stress is placed on the joints of the structures, which can lead to premature failure.

If accurate control and stress reduction are not relevant issues for a given application, the engine can be operated at high frequencies with simple periodic signals such as sine or square waves. Otherwise, modelbased drive signals should be used to achieve optimum performance.

\subsubsection{Stopping and Starting}

The linear inertia of the moving electrostatic comb drives can have a significant impact on the process of starting and stopping the microengine. We examine what happens when high speed operation of the microengine is abruptly stopped by simply turning off the voltage driving the linear actuators.

Consider the case where the engine is running at a constant speed well above the resonant frequency. In this regime, the electrostatic force that accelerates the shuttle is very large compared to friction and restoring spring forces alone. Recall from the previous discussions that when the correct drive signal is used, the force at the gear pin joint can be made on the order of $0.1 \mathrm{kr}$. The force provided by the $y$ comb drive at speeds above the resonant frequency can easily exceed $5 \mathrm{kr}$, a factor of 50 greater than the force experienced by the pin joint. Thus, if the engine voltage is turned off while the engine is running, the inertial force, which is normally provided electrostatically, will be provided directly by the pin joint connecting the gear to the link arm. This force can exceed the normal operating force experienced by the pin joint by more than a factor of 50 , causing premature failure. The actual magnitude of the force depends on both the speed at which the engine is running and its angular position when it is abruptly turned off. A similar type of situation occurs when power is disconnected.

The excessive stress associated with abrupt starting and stopping can be mitigated by using properly engineered drive signals during these transients. This can be done by letting the instantaneous speed $d \theta / d t$ in Eqs. (7) and (8) ramp quickly with time. For example, by letting

$$
\begin{gathered}
\theta(t)=\omega t-\frac{1}{2} \alpha t^{2} \\
\dot{\theta}(t)=\omega-\alpha t \\
\ddot{\theta}(t)=-\alpha
\end{gathered}
$$

with $\omega=10500 \mathrm{rad} / \mathrm{s}$ and $\alpha=3 \times 10^{6} \mathrm{rad} / \mathrm{s}^{2}$, the engine will ramp from 100,000 rpm to a complete stop in just 3 revolutions of the output gear. This rapid deceleration does not result in excessive stress in the pin joint, since all of the relevant forces are compensated by the modelbased drive signals. The primary trade-off to be considered here involves the acceleration rate; the greater the acceleration, the greater the voltage required during the ramp in speed. 
The impact of start/stop signals on engine performance is experimentally observed to be quite dramatic. Engines operated well above 100,000 rpm typically fail after on the order of a dozen start/stop cycles when improper start/stop signals are employed. Using ramps as shown in Eq. 10, we have achieved a greater than 3 order of magnitude improvement in start/stop endurance of the engine. In one experiment, while accruing 3.2 billion revolutions at a speed of $146,000 \mathrm{rpm}$, an engine experienced over 66,000 start/stop cycles. This feat was achieved with no lubrication. It is clear that engine performance is dramatically improved by properly taking into account inertial forces, both when operating at constant speeds as well as during stopping and starting.

The inertial analysis and corresponding experimental results enable a designer or end user of the microengine to make accurate and relevant performance tradeoffs. Optimum performance is clearly achieved using model-based drive signals. Using the correct signals, the engine can be started and stopped very quickly - typically within a few revolutions of the gear. If an end user is constrained to use conventional signals, such as sine waves, the inertia-related stop/start stress can be reduced simply by making speed changes occur more slowly, such as over many cycles. This also permits the inertia related phase shift of the gear to occur smoothly.

\subsection{Viscous Damping}

Viscous damping forces arise from the mechanical components moving through air or some other viscous medium, such as oil. To achieve proper operation of the engine, these forces must be overcome by the application of appropriate drive signals. We now

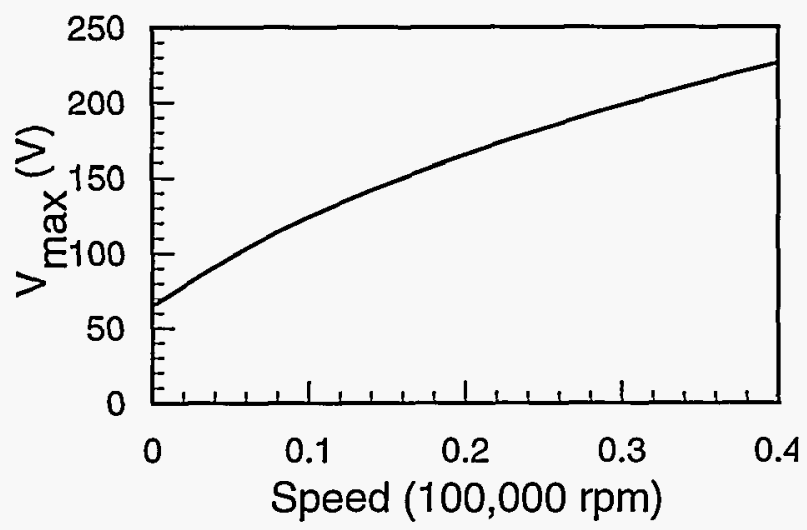

Fig. 9. The voltage required to operate the engine with significant damping increases as the square root of the speed (compare with Fig. 5 for low viscous damping). Note that the speed scale here differs by an order of magnitude from that in Fig. 5. consider two cases: damping in air and damping in oil.

In air, the damping coefficient $\delta$ for the engine has been measured to be $2300 \mathrm{rad} / \mathrm{sec}^{6}$ Consequently, the damping force for air even at $100,000 \mathrm{rpm}$ is only $\sim 0.4 \mathrm{kr}$. At lower speeds, air damping forces become much smaller than spring forces. At higher speeds, inertial forces dominate. Thus, while air damping impacts the performance of resonant structures by lowering their quality factor, the effects of air damping on engine operation can typically be ignored. Air damping forces are non-trivial only near the resonant frequency, and even then it is not dominant.

In contrast, the damping force due to oil can be significant, even at very low speeds. For an engine operating in silicone oil (2 CS), we measured the damping coefficient to be $\delta \sim 3 \times 10^{5} \mathrm{rad} / \mathrm{s}$. This is a factor of 130 greater damping than that for operation in air. Thus, the damping force becomes comparable with the spring force $k r$ at a speed of only $\sim 2000 \mathrm{rpm}$. Due to the large damping coefficient, significant drive voltages are required to achieve high speeds. To illustrate, the peak voltage in the $y$ drive signal required to operate the engine is shown in Fig. 9 as a.function of engine speed. The drive voltage increases as the square root of the engine speed. The phase of the drive signal also changes quickly with speed, as seen in Fig. 10. The impact of viscous damping on model-based drive signals is further seen by comparing Figs. 9 and 10 (oil damping) with Figs. 5 and 6 (air damping). Note that the speed scales for Figs. 5 and 6 differ by an order of magnitude from those of Figs. 9 and 10.

The ability of model-based drive signals to properly compensate for viscous damping effects is experimentally validated by the data shown in Fig. 11 . An engine is operated in oil at a range of speeds. At the

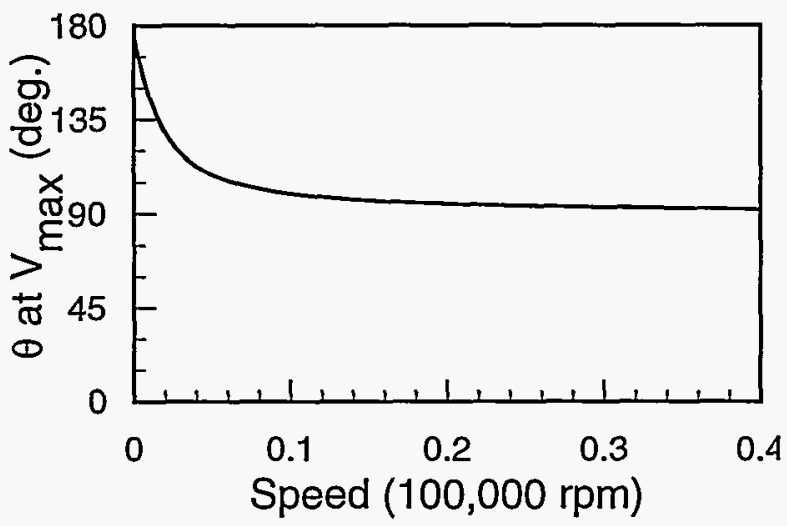

Fig. 10. For significant damping, the phase of the peak in the drive voltage quickly asymptotes to $90^{\circ}$, in contrast with $0^{\circ}$ for the case of negligible damping (see Fig. 6). 


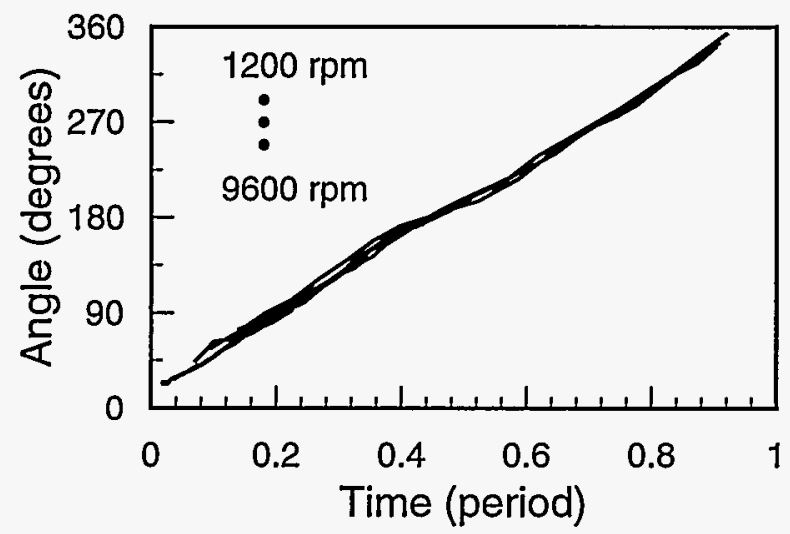

Fig. 11. An engine is operated in oil using drive signals created with Eqs. (7) and (8), using the experimentally measured damping coefficient $\delta$. Data are shown for engine speeds of $1200,2400,4800,6600$, and $9600 \mathrm{rpm}$. At the top speed of $9600 \mathrm{rpm}$, the viscous damping force is nearly 6 times the spring force.

lowest speed (1200 rpm) the peak damping force is $\sim 0.7 \mathrm{kr}$. At the top speed $(9600 \mathrm{rpm})$, the peak damping force is nearly $6 \mathrm{kr}$, more than a factor of 8 higher. As shown by the data, the engine responds properly for this wide range of speeds and damping forces. The lack of a phase shift between the data for different speeds (selfadjustment) indicates that the forces are properly accounted for by the model-based drive signals.

The performance model not only can be used to achieve optimum position control, as demonstrated by the data in Fig. 11, but also can be used to make performance tradeoffs. For example, viscous lubricants may be employed to reduce friction and wear for some low-speed applications. Depending on the required operational speed, the performance model can be used to accurately determine the maximum viscosity lubricant that can be used before the drive signal amplitude is significantly impacted.

\subsection{Load Inertia}

When accelerating inertial loads, such as a $1.6 \mathrm{~mm}$ diameter optical shutter shown in Fig. $12,1,2$ the microengine converts electrical energy into kinetic energy. This transfer of energy requires an amount of time that depends on the magnitude of the torque being applied to the load. If the torque history delivered by the engine is not properly chosen, the load gear will not accelerate, and the engine will simply vibrate. The load cannot respond fast enough if the drive frequency is too high.

Loads with inertia can be controlled using modelbased drive signals. To accelerate a load with rotational inertia requires a load force (in addition to that required to overcome friction) of

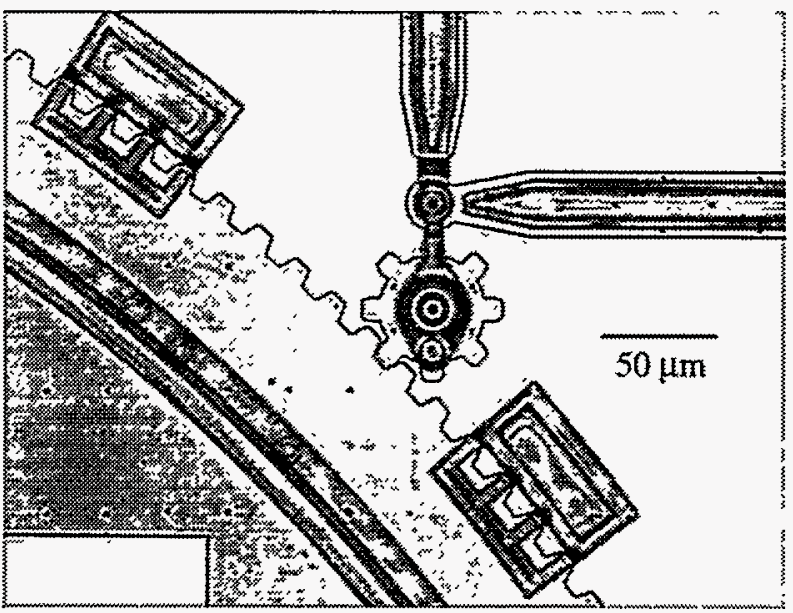

Fig. 12. Rapidly accelerating an inertial load, such as the 1.6 $\mathrm{mm}$ diameter optical shutter shown in the lower left, requires a properly tailored torque history provided by the performance model.

$$
\frac{F_{l}}{k r}=\frac{\ddot{\theta}}{k} \frac{I_{2}}{R_{2}^{2}}\left(\frac{R_{1}}{r}\right)^{2}
$$

The previously undefined terms occurring in Eq. (11) are specified in Fig. 13. We note that the rotational inertia of the $50 \mu \mathrm{m}$ diameter engine drive gear does not occur in the equations, since associated forces are small compared to inertial forces of the combs.

The magnitude of the inertial force given by Eq. (11) is illustrated with a practical example. In order to accelerate the optical shutter from rest to a speed of $4500 \mathrm{rpm}$ in one rotation of the engine drive gear, an additional load force of $F_{l} / k r \sim 3$ is needed. If the ramp in speed is needed to occur in an even shorter interval, a much larger force will be necessary, requiring a much

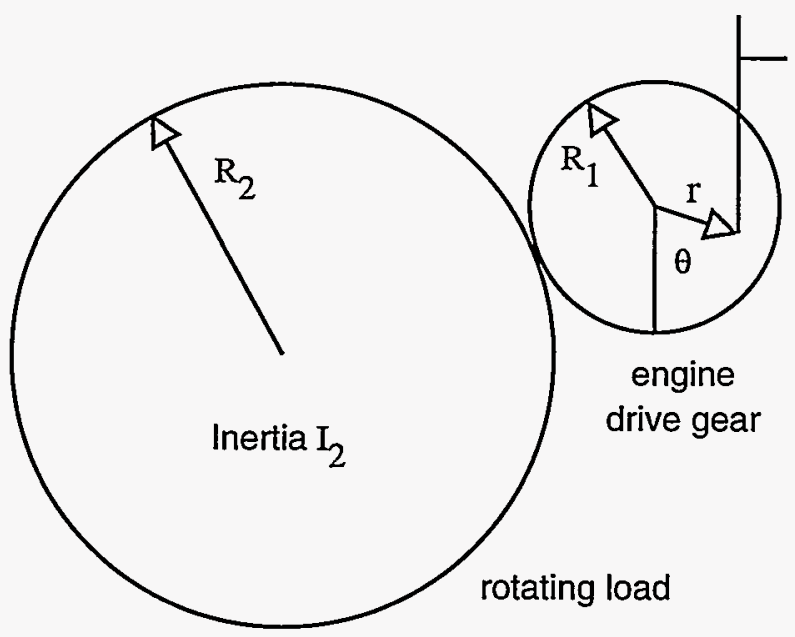

Fig. 13. The small engine gear drives a large rotating load gear with inertia. The rotational inertia of the engine gear results in negligible forces compared to others in the system. 
higher drive voltage. Model-based drive signals created by combining Eq. (11) with Eqs. (7) and (8) result is the proper torque history to achieve the desired acceleration.

If model-based drive signals are not used to accelerate the load gear, several things will occur. First, the position of the gear will not be in its expected position because the system will self-adjust to compensate for an improper electrostatic force history. This will result in increased frictional forces and linkage stress, as discussed in previous sections. Secondly, if energy is not transferred to the load at a sufficiently high rate, the oscillation rate of the drive signals will exceed the rate at which the drive gear will turn. When this occurs, the engine will simply vibrate in response to the drive signal, but the load will not rotate. A similar situation occurs when driving non-rotating inertial loads, such as a linear rack.

We have obtained experimental verification of the impact of load inertia on acceleration rate. When square wave drive signals are abruptly applied to the engine, the optical shutter (Fig. 12) will begin rotating for attempted speeds less than $\sim 600 \mathrm{rpm}$ (shutter speed). The engine system compensates for the non-ideal drive signals by self-adjusting its phase. For attempted speeds above $\sim 600 \mathrm{rpm}$, the load gear will not start rotating; the engine simply vibrates as described above. However, by using model-based drive signals, shutter speeds of 4500 $\mathrm{rpm}$ (engine speeds of 146,000 rpm) have been achieved. This is nearly an order of magnitude improvement in speed of an inertial load.

The primary performance tradeoffs to be made while driving inertial loads pertain to the acceleration rate. If the microengine must achieve a large load acceleration, the comb drives actuating the engine gear must be properly designed to provide sufficient force for the available drive signal amplitude. If high acceleration rates of inertial loads are not needed, the additional force contribution given by Eq. (11) is negligible. If phasing and friction are not an issue, slowly varying sine or square wave drive signals can be used to actuate inertial loads with the microengine.

\section{Summary}

The two-fold objective of this work has been achieved, namely 1) understand how various physical quantities impact performance in a range of operational scenarios, and 2) quantify performance tradeoffs. We have developed a model of the microengine system that accommodates the dominant forces affecting the system. These forces originate from 1) support springs, 2) friction between the gear and hub, 3) linear inertia of the comb drives, 4) viscous damping, and 5) load inertia. We have demonstrated how to create modelbased drive signals to operate the engine to achieve desired performance. Using these drive signals, we have demonstrated controlled operation of the engine where each of the above 5 forces are dominant. In doing so, we have experimentally validated methods to minimize friction, minimize joint stress, maximize angular acceleration, and achieve precise positioning of the output gear.

Using model-based drive signals, we have demonstrated reduced frictional forces $(>10 \mathrm{x}$ improvement), demonstrated prolonged high speed operation ( 3.2 billion cycles at $146,000 \mathrm{rpm}$ ) where inertial forces are dominant $(>10 \mathrm{x}$ improvement), demonstrated over 66,000 high speed start/stop cycles $(>1000 \mathrm{x}$ improvement), demonstrated controlled operation in viscous damping media where viscous forces dominate, and demonstrated acceleration of inertial loads to $4600 \mathrm{rpm}$ (nearly $10 \mathrm{x}$ improvement).

The results presented in this paper enable both designers and end users of microengine-powered systems to make accurate and relevant performance tradeoffs. When these tradeoffs are intelligently made, the result will be actuator systems whose performance is truly optimized. These results significantly enhance the value of the microengine as a controllable micromechanical power source.

\section{Acknowledgments}

The authors are grateful to Michael Callahan for funding this work, and the personnel of the Microelectronics Development Laboratory at Sandia National Laboratories for fabricating the devices used in this study.

This work was performed at Sandia National Laboratories and supported by the U. S. Dept. of Energy under contract DE-AC04-94AL85000.

\section{References}

1. J. J. Sniegowski and E. J. Garcia, "SurfaceMicromachined Gear Trains Driven by an On-Chip Electrostatic Microengine", IEEE Electron Device Lett., vol 17, pp. 366-368, 1996.

2. J. J. Sniegowski, S. L. Miller, G. LaVigne, M. S. Rodgers, and P. J. McWhorter, "Monolithic Geared-Mechanisms Driven by a Polysilicon Surface-Micromachined On-chip Electrostatic Engine", Technical Digest of the 1996 Solid State Sensor and Actuator Workshop, Hilton Head Island, SC, June 3-6, 1996, pp. 178-182.

3. J. J. Sniegowski and E. J. Garcia, "Microfabricated actuators and their application to optics", Proc. SPIE Miniaturized Systems with Micro-Optics and Micromechanics, Vol. 2383. San Jose, CA, Feb. 79, 1995, pp. 46-64. 
4. Technology of the Year award, Industry Week, A Penton Publication, Vol 244, No. 23, Dec 18, 1995, pp. 30-34.

5. E. J. Garcia and J. J. Sniegowski, "Surface micromachined microengine", Sensors and Actuators A, Vol. 48, (1995) pp. 203-214.

6. S. L. Miller, J. J. Sniegowski, G. LaVigne, and P. J. McWhorter, "Friction in Surface Micromachined Microengines", Proc. SPIE Smart Electronics and MEMS Vol. 2722, San Diego, Feb. 28-29, 1996, p. 197-204. 